\title{
Does An Orthodontic Functional Appliance Correct Head and Cervical Spine Posture
}

\author{
Hoger Omran Alsheikho ${ }^{1 *}$, Batoul Ali Mohammad ${ }^{2}$ and Farah Harba ${ }^{2}$ \\ ${ }^{1}$ Department of Orthodontics, Faculty of Dentistry, Tishreen University, Syria \\ ${ }^{2}$ Faculty of Dentistry, Tishreen University, Syria
}

*Corresponding author: Hoger Omran Alsheikho, Department of Orthodontics, Faculty of Dentistry, Tishreen University, Alzera, Latakia, Syria.

To Cite This Article: Hoger Omran Alsheikho, Batoul Ali Mohammad, Farah Harba. Does An Orthodontic Functional Appliance Correct Head and Cervical Spine Posture. Am J Biomed Sci \& Res. 2021 - 13(2). AJBSR.MS.ID.001845. DOI: 10.34297/AJBSR.2021.13.001845.

Received: 眥 June 04, 2021; Published: 眥 June 10, 2021

Keywords: Functional Appliance; Head Posture; Cervical Spine Posture

\section{Opinion}

Class II malocclusion is one of the most common orthodontic problems, it is observed in one -third of the general population [1]. It is mostly diagnosed due to retrognathia of the mandibular. Functional appliances are considered orthodontic appliances that have dental, alveolar, and skeletal effects. It influences the patient's facial skeletal component during growth. Orthodontic functional appliances are usually used to correct mandibular retrognathia accompanying skeletal class II malocclusion [2].

Many researchers have studied the effect of orthodontic functional appliances on the head and spinal column posture [3-7]. Tecco et al. [3] evaluated the cervical spine posture after treatment with functional regulator Frankel II, and observed changes in the middle and lower segments of the cervical spine. But the change in the observed angle was small 2-3 ${ }^{\circ}$. Meanwhile Ohnmei $\beta$ et al [4] studied the treatment effect on the posture of the cervical spine using 'Activator' and 'Bite Jumper' appliances, and noticed a single change in the variables that was in the (CVT/FH) angle, whilst the rest of the variables did not show any evidence of a change in the posture of the head. On the other hand, the only statistically significant change that was observed by Aglarci [5] who used 'Twin Block' appliance to study the posture was in the (OPT/CVT) angle $(\mathrm{p}=0.009)$. Kamal also studied the head and cervical spine posture by using a the 'Twin Block' appliance, the only observed change was a 3-degree increased in the (SN/OPT) angle of the upper cervical spine from -3.5 to $0.5(\mathrm{p}=0.032)$ [6]. Hoger et al. [7] studied another appliance 'Bionter' and 'Twin Block', but it did not observe any change in of the angles that were studied, i.e. that the treatment using functional appliances did not cause any changes in the posture of the head and cervical spine.

Looking at the previous studies we can notice the aside from Tecco's trial, all researches only observed one statistically significant change in the studied variables. The reason for this minimal change observed in these articles could be attributed to the fact that the mandibular jaw is not separated from the rest of the body, as it is connected to multiple muscles, bones, and ligaments. Functional appliances only focus its force on the upper and lower jaws, whilst the surrounding ligaments and muscles have to adapt to the new positioning.

One of other components that might contribute to the lack of posture changes is the hyoid bone which is connected to two main muscle groups (Infrahyoid and suprahyoid) [8]. To illustrate this Thurow [9] found that when the head is extended, the hyoid bone is pushed forward by the paasive extension of the suprahyoid muscles, this is similar to the mechanism observed in patients who are forced to breathe through the mouth, as the very crucial cranial extension makes up for the nasal airway obstruction. The suprahyoid muscles play an important role in cranial balance, and also they are connected to the hyoid bone, the larynx, the pharynx 
and the tongue. And because of these connections near or at the symphysis of the lower jaw, the movement of the hyoid bone is consistent with the movement of the chin, and movements from all the soft tissues would have an effect on the oropharyngeal airway [10]. Furthermore, hyoid muscle fibers, and the stylohyoid ligament serve as rigging lines that define the hyoid bone's range of movement; During head extension, the hyoid bone moves backward, and during head flexion, the hyoid bone moves forward whilst maintaining its level as much as possible. In addition, the hyoid bone moves a little backward when the mouth opens [11]. In addition, the fact that the hyoid bone is not affected by the functional treatment could be one of the factors that prevent changes in the head and cervical spine positioning in order to maintain the patency of the airway and to help the patient breathe efficiently.

Another important factor that is considered responsible for the lack of change is the neuromuscular junction of the spinal cord. As the sternocleidomastoid muscle is considered the main contributing muscle in the balance of the head [12]. Valenzuela et al [13] observed a lack of change in the sternocleidomastoid muscle activity despite changed head posture, which could have been due to the functional demands of the patient's face and the ability to manage optic vision directed horizontally.

Unchanged activity of the sternocleidomastoid muscle was found to play a major role in the unchanged posture, that's because neurons from cranial nerves (V, VII, IX, X) share the same neuron pool with neurons from the upper segments of the cervical spine $[14,15]$. In addition, trigeminal inputs must be taken into consideration, and that is because of the important relationship between the descending tract of the trigeminal nerve and the upper dorsal roots. It has been well documented that trigeminal influences play a role in the neuromuscular program during habitual occlusion [16]. All of this leads to the conclusion that trigeminal inputs from the periodontia, temporomandibular joint, and from muscular receptors might be contributing to the modulation of the motor neurons pool of the sternocleidomastoid muscles. Thus, to have any change in posture, a change must be first made in the neuromuscular junction, trigeminal inputs and the sternocleidomastoid muscle.

In conclusion, whilst functional appliances cause a statistically significant change in the posture of the head and the cervical spine, this change is not enough for us to consider these appliances as stature correctors (Head, Spinal cord, Body), and this can be contributed to the need of appliances that do not only rely on the maxilla and the mandible, but rely on the cervical spine and the head, and this way it can indirectly move the mandible, and change the positioning of the head.

\section{References}

1. Proffit WR, Fields HW, Moray LJ (1998) Prevalence of malocclusion and orthodontic treatment need in the United States: estimates from the N-HANES III survey. Int J Adult Orthod Orthog Surg 13(2): 97-106.

2. McNamara JA Jr, Brudon WL (2001) Orthodontics and dentofacial orthopedics. Ann Arbor: Needham Press p. 67-80.

3. Tecco S, Farronato G, Salini V, Di Meo S, Filippi MR, et al. (2005) Evaluation of cervical spine posture after functional therapy with FR 2: A longitudinal study. Cranio 23(1): 53-66.

4. Ohnmeiß M, Kinzinger G, Wesselbaum J, Korbmacher-Steiner HM (2014) Therapeutic effects of functional orthodontic appliances on cervical spine posture: a retrospective cephalometric study. Head Face Med 10:

5. Aglarci C (2016) Evaluation of cervical spine posture after functional therapy with twin-block appliances. J Orthod Res 4(1): 6-12.

6. Kamal A, Fida M (2019) Evaluation of cervical spine posture after functional therapy with twin- block appliance: A Retrospective Cohort Study. Am J Orthod Dentofacial Orthop 155(5): 654-661.

7. Hoger Al, Doaa J, Mohamad Y, Mohammed T,Hazem H, et al. (2021) Evaluation of head and cervical spine posture after functional therapy with Twin-Block and Bionator appliances: A pilot randomized controlled trial. Cranio 11:1-10.

8. Goss CM (1967) Anatomy of the Human Body. $28^{\text {th }}$ edition. Philadelphia: Lea and Febiger.

9. Thurow RC (1978) Atlas of Orthodontic Principles. St. Louis: CV Mosby.

10. Graber LW (1978) Hyoid changes following orthopedic treatment of mandibular prognathism. Angle Orthod 48: 33-38.

11. Ingervall B, Carlsson GE, Helkimo M (1970) Change in location of hyoid bone with mandibular positions. Acta Odontol Scand; 28(3): 337-361.

12. Weeks DV, Travell J (1955) Postural vertigo due to trigger areas in the sternocleidomastoid muscle. J Pediatr 47(3): 315-327.

13. Valenzuela S, Miralles R, Ravera M J, Zúñiga C, Santander H, et al. (2005) Does Head Posture Have a Significant Effect on the Hyoid Bone Position and Sternocleidomastoid Electromyographic Activity in Young Adults? Cranio 23(3): 204-211

14. Green JD, De Groot J, Sutin J (1957) Trigemino-bulbar reflex pathways. Am J Physiol 189(2): 284-288.

15. Sumino R, Nozaki S (1977) Trigemino-neck reflex: its peripheral and central organisation. In: Anderson DJ, et al (Eds.)) Pain in the trigeminal region. Amsterdam pp. 365-375.

16. Laat A (1987) Reflexes elicitable in jaw muscles and their role during jaw function and dysfunction: A Review of the Literature. Part III. Reflexes in human jaw muscles during function and dysfunction of the masticatory system. Cranio 5(4): 333-343. 